\title{
Sintomas de depressão pós-parto e sua associação com as características socieconômicas e de apoio sociala
}

\author{
Postpartum depression symptoms and association with socieconomic and social support characteristics \\ Síntomas de la depresión posparto y su asociación con características socioeconómicas y de apoyo \\ social
}

\author{
Maria Luiza Cunha Santos ${ }^{1}$ (1) \\ Joyce Ferreira Reis ${ }^{1}$ (1) \\ Ranielle de Paula Silva² (1) \\ Dherik Fraga Santos ${ }^{2} \mathbb{C}$
}

Franciéle Marabotti Costa Leite ${ }^{2}$ (D)

1. Universidade Federal do Espírito Santo, Departamento de Enfermagem. Vitória, Espírito Santo, Brasil.

2. Universidade Federal do Espírito Santo, Programa de Pós-Graduação em Saúde Coletiva. Vitória, Espírito Santo, Brasil.
Autor Correspondente:

Franciéle Marabotti Costa Leite.

E-mail: francielemarabotti@gmail.com

Submitted on $07 / 01 / 2021$.

Accepted on 12/06/2021.

\begin{abstract}
Resumo
Objetivo: verificar a prevalência de sintomas de depressão pós-parto em puérperas atendidas em uma maternidade pública e sua associação com características socioeconômicas e de apoio social. Método: estudo epidemiológico, analítico, do tipo transversal, em uma maternidade pública conduzido de agosto a outubro de 2017. A amostra de 330 puérperas foi entrevistada por meio da aplicação de um formulário, para mensuração da presença de sintomas de depressão pós-parto. Foi utilizada a escala de depressão pós-natal de Edimburgo. Já para mensuração do apoio social, foi utilizado o instrumento Medical Outcomes Study. A medida de associação adotada foi a razão de prevalência (RP) com intervalos de confiança de $95 \%$ (IC95\%), e aplicada a regressão de Poisson ajustada. Resultados: a prevalência de sintomas de DPP foi de 29,7\%. A idade entre $14 \mathrm{e}$ 24 anos (PR:1,60; 95\%Cl: 1,10-2,34), ter até 8 anos de escolaridade (RP:1,39; IC95\%:1,01-2,14) e o baixo nível de suporte social afetivo (RP:1,52; IC95\%:1,07-2,14) e emocional (RP:2,12; IC95\%:1,41-3,19) estiveram associados à maior prevalência de sintomas de DPP. Conclusão e implicações para a prática: nesse contexto, os profissionais de saúde podem possuir um papel essencial no qual podem desenvolver, em conjunto, um plano de cuidados de acordo com as necessidades da mulher em período gravídico-puerperal.
\end{abstract}

Palavras-chave: Apoio Social; Depressão Pós-Parto; Fatores de Risco; Período Pós-Parto; Saúde da Mulher.

\begin{abstract}
Objective: to verify the prevalence of postpartum depression symptoms in postpartum women assisted at a public maternity hospital and its association with socioeconomic and social support characteristics. Method: this is an epidemiological, analytical, cross-sectional study in a public maternity hospital conducted from August to October 2017. A sample of 330 postpartum women was interviewed using a form to measure the presence postpartum depression symptoms. The Edinburgh Postnatal Depression Scale was used. To measure social support, the Medical Outcomes Study instrument was used. The measure of association adopted was the prevalence ratio (PR) with $95 \%$ confidence intervals $(95 \% \mathrm{Cl})$, and adjusted Poisson regression was applied. Results: the prevalence of PPD symptoms was $29.7 \%$. Age between 14 and 24 years (PR:1.60; 95\%Cl: 1.10-2.34), have up to 8 years of education (PR:1.39; 95\%Cl: 1.01-2.14) and the low level of affective (PR:1.52; 95\%Cl: 1.07-2.14) and emotional (PR:2.12; $95 \% \mathrm{Cl}: 1.41-3.19)$ social support were associated with higher prevalence of PPD symptoms. Conclusion and implications for practice: in this context, health professionals can play an essential role in which they can jointly develop a care plan according to the needs of women in the pregnancy-puerperal period.
\end{abstract}

Keywords: Depression Postpartum; Postpartum Period; Risk Factors; Social Support; Women's Health.

\section{Resumen}

Objetivo: verificar la prevalencia de síntomas de depresión posparto en mujeres posparto atendidas en una maternidad pública y su asociación con características socioeconómicas y de apoyo social. Método: estudio analítico transversal en una maternidad pública realizada entre agosto y octubre de 2017. La muestra de 330 puérperas fue entrevistada mediante la aplicación de un formulario, para medir la presencia de síntomas de depresión postparto. Se utilizó la Escala de Depresión Postnatal de Edimburgo. Para medir el apoyo social se utilizó el instrumento Medical Outcomes Study. La medida de asociación adoptada fue la razón de prevalencia (RP) con intervalos de confianza del 95\% (IC del 95\%) y se aplicó la regresión de Poisson ajustada. Resultados la prevalencia de síntomas de DPP fue de 29,7\%. Edad entre 14 y 24 años (PR:1,60; 95\%Cl: 1,10-2,34), tener hasta 8 años de escolaridad (RP: 1,39; IC 95\%: 1,01 - 2,14) y el bajo nivel de apoyo social afectivo (RP: 1,52; IC del 95\%: 1,07-2,14) y emocional (RP: 2,12; IC del 95\%: 1,41-3,19) se asociaron con una mayor prevalencia de síntomas de PPD. Conclusión e implicaciones para la práctica: en este contexto, los profesionales de la salud pueden jugar un papel fundamental en el que puedan desarrollar conjuntamente un plan de cuidados acorde a las necesidades de la mujer en el período gestacional-puerperal.

Palabras clave: Apoyo Social; Depresión Posparto; Factores de Riesgo; Periodo Posparto; Salud de La Mujer. 


\section{INTRODUÇÃO}

A depressão é um transtorno mental frequente, que acomete cerca de 300 milhões de pessoas. Corresponde a uma das principais causas de incapacidade em todo o mundo. Caracteriza-se pela presença de humor depressivo, perda de interesse ou prazer em quase todas as atividades, ${ }^{1}$ que levam à necessidade de cuidados, e, consequentemente, aos rearranjos cotidianos para provê-los. ${ }^{2}$

No Brasil, cerca de $15,5 \%$ dos brasileiros são afetados pela depressão em algum momento da vida. ${ }^{3}$ As mulheres vivenciam a depressão 1,5 a 3 vezes mais transtornos depressivos, ${ }^{4}$ sendo que, no puerpério, fase em que ocorrem diferentes transformações fisiológicas e psíquicas, há a possibilidade da ocorrência da depressão pós-parto (DPP). ${ }^{5}$

A DPP é uma condição de profunda tristeza, desespero, falta de esperança, melancolia, desmotivação, ausência de forças para lidar com a rotina, ${ }^{6}$ que pode ocorrer por alguns dias ou até meses depois do parto. ${ }^{7}$ A experiência da DPP pode gerar na puérpera o medo, a dúvida e a angústia em relação à capacidade de cuidar do bebê e às transformações no estilo de vida, podendo acarretar efeitos negativos à saúde da mulher. ${ }^{8}$

Nos Estados Unidos, verifica-se uma prevalência de 2,5\% de sintomas de DPP entre puérperas, ${ }^{9}$ enquanto na República Tcheca foi observada uma prevalência de 11,8\%. ${ }^{10}$ Estudo realizado no Egito apresentou $7 \%$ dessa frequência ${ }^{11}$. Em países da América Latina, o Chile mostrou uma prevalência de $21 \%$ de sintomas de DPP. ${ }^{12}$ Já no Brasil, diferentes estudos já observaram importantes oscilações da prevalência, indo de 6,7\% em São Paulo ${ }^{13}$ a $25,8 \%$ em estudo nacional. ${ }^{14}$

Diversas variáveis podem estar relacionadas à ocorrência dos sintomas de DPP, como ter idade jovem, não ter parceiro, uso de tabaco antes da gestação, consumo de bebida alcóolica e história de aborto. ${ }^{15} \mathrm{~A}$ vivência de violência na gestação, parto por cirurgia cesárea, ${ }^{13}$ história familiar de depressão e menor escolaridade ${ }^{16}$ também se mostram importantes na ocorrência da DPP.

A mulher com DPP necessita de apoio social, ${ }^{17}$ visto que o suporte social possui relação direta com o aumento na capacidade de enfrentar situações difíceis. ${ }^{18}$ Assim, a presença e o apoio de familiares, do parceiro e amigo pode contribuir para uma experiência positiva da gravidez. ${ }^{19}$ Receber apoio, afeto, cuidado e proteção na gestação é fundamental para que o período gravídico puerperal transcorra com tranquilidade. ${ }^{20}$

É importante destacar que, quanto antes a DPP for identificada, maiores as chances de prevenir os danos, ${ }^{16}$ como a diminuição do vínculo mãe-bebê e os atrasos no desenvolvimento social e cognitivo dos filhos. ${ }^{6}$ Dessa maneira, os profissionais de saúde podem avaliar o risco entre as puérperas e prestar os cuidados adequados para evitar que a depressão se desenvolva ou, no caso de já instalada, não se agrave. ${ }^{21}$

Diante do exposto, considerando a magnitude da DPP e o impacto negativo na saúde do binômio, bem como a importância de se identificar a realidade de cada local, este estudo visa verificar a prevalência de sintomas de DPP em puérperas atendidas em uma maternidade pública e sua associação com características socioeconômicas e de apoio social.

\section{MÉTODOS}

\section{Tipo de estudo}

Estudo epidemiológico, analítico do tipo transversal, realizado em uma maternidade pública do município de Cariacica, Espírito Santo.

\section{Contexto}

Trata-se de uma maternidade de referência no atendimento à gestação de baixo risco, na qual são ofertados serviços ambulatoriais, atendimento a urgências e emergências obstétricas 24 horas e internação. Para tal, 45 leitos de enfermaria obstétrica e quatro de pré-parto são destinados. Essa maternidade é gerenciada por uma instituição filantrópica, e todos os atendimentos são realizados pelo Sistema Único de Saúde (SUS), onde o custeio é feito pelo Governo do Estado, por meio da Secretaria de Estado da Saúde. O município de estudo está localizado na Região Metropolitana da Grande Vitória, possui 381.285 habitantes com índice de desenvolvimento urbano municipal de $0,718 .{ }^{22}$

\section{Participantes}

Como critério de inclusão, adotou-se ser puérpera internada em uma maternidade pública do município de Cariacica com, no mínimo, 24 horas de pós-parto, de feto vivo (acima de 500 gramas) independente da via de parto e ter tido parceiro íntimo durante a gestação. Já os critérios de exclusão, foram puérperas com déficit de audição, linguagem, cognitivo ou quadro de demência que possa prejudicar ou impedir, de alguma forma, o devido entendimento do estudo.

\section{Fontes de dados e mensuração}

As entrevistas ocorreram entre agosto e outubro de 2017, em espaço privativo, sendo permitida apenas a presença do recémnascido. Primeiramente, as puérperas foram informadas sobre o objetivo do estudo e após o aceite e assinatura do Termo de Consentimento Livre e Esclarecido (TCLE) acontecia a entrevista, sendo que as participantes menores de 18 anos assinaram o Termo de Assentimento frente a assinatura do TCLE pelo seu responsável. As características socioeconômicas das puérperas foram obtidas por meio de formulário próprio, em que foram coletadas as seguintes informações: idade; situação conjugal; escolaridade; classe econômica da Associação Brasileira de Empresas de Pesquisas (ABEP).

\section{Variáveis em estudo}

A fim de identificar a escala de apoio social, foi usado o instrumento Medical Outcomes Study (MOS), validado em português, que pretende avaliar as principais dimensões do apoio social de modo prático e breve ${ }^{24} \mathrm{~A}$ escala possui cinco dimensões avaliadas (apoio emocional, apoio informacional, interação social, apoio material e apoio social afetivo). O apoio 
material diz respeito ao fornecimento de recursos práticos e ajuda material; ao afetivo, a demonstrações físicas de amor e afeto; à interação social positiva, a contar com pessoas com quem relaxar e se divertir; ao emocional, à capacidade da rede social de suprir as necessidades individuais como problemas emocionais que exigem sigilo; e ao âmbito da informação, que representa contar com pessoas que informem, aconselhem $e$ orientem. ${ }^{25}$

No presente estudo, o apoio social foi dividido em cinco dimensões: material (baixo, alto); afetivo (baixo, alto); emocional (baixo, alto); de informação (baixo, alto); e de interação positiva (baixo, alto). Classificou-se como alto ou baixo de acordo com a média de cada dimensão. Dessa forma, as mulheres que pontuaram abaixo da média das respectivas dimensões tiveram a classificação de baixo apoio e aquelas que estavam acima da média são pontuadas com alto nível de apoio social.

Para a identificação do desfecho e sintomas de DPP, foi aplicado o instrumento da Escala de Depressão Pós-Natal de Edimburgo (EPDS), validado em português, que possui dez itens. Cada item é somado aos demais itens para obtenção da pontuação total, que varia de 0 a 30 pontos. ${ }^{26}$ Para a pesquisa, dos sintomas de DPP, foram considerados presentes quando a mulher atingia uma pontuação maior ou igual a 12. Dessa maneira, as puérperas que pontuaram abaixo de 12 foram categorizadas como não.

Para o rastreamento dos sintomas de DPP, desfecho em estudo, foi utilizado o instrumento intitulado EPDS. Essa variável dependente foi analisada de forma dicotômica (sim, não).

As variáveis independentes incluíram:

a) Idade (em anos: 14-24, 25 ou mais)

b) Situação conjugal (casada/união consensual, solteira/ namorando)

c) Escolaridade (em anos: até 8, 9 ou mais)

d) Classe econômica ABEP (B/C, D/E)

e) Apoio social/material (baixo, alto)

f) Apoio social/afetivo (baixo, alto)

g) Apoio social/emocional (baixo, alto)

h) Apoio social/informação (baixo, alto)

i) Apoio social/interação social positiva (baixo, alto)

\section{Controle de viés}

Para prevenir a ocorrência de viés do observador, as entrevistadoras do estudo participaram de um treinamento, com o objetivo de padronizar a realização das entrevistas e a aplicação dos formulários em período prévio à coleta de dados. O treinamento dos entrevistadores foi realizado no mês de julho de 2017, sendo contabilizada uma carga horária de 20 horas. No treinamento, as entrevistadoras foram capacitadas para aplicação dos instrumentos e orientadas quanto aos aspectos éticos, à neutralidade, à privacidade e ao sigilo durante todo o processo da coleta de dados. O teste piloto foi realizado com 50 puérperas. As entrevistadoras foram acompanhadas pelo pesquisador responsável pela pesquisa e avaliadas quanto à abordagem à mulher, habilidade e aplicação correta dos instrumentos. As entrevistas realizadas nesse período não fizeram parte da amostra selecionada no presente estudo.

\section{Amostragem}

Para o cálculo da amostra de estudo, foi considerada a prevalência do desfecho em $50 \%,{ }^{23}$ nível de confiança de $95 \%$ e margem de erro de $5 \%$. O tamanho da amostra foi 235 participantes. Acrescidos de $10 \%$ de perda e $30 \%$ para fatores de confusão, o tamanho da amostra foi de 330 puérperas.

\section{Métodos estatísticos}

Os dados foram digitados na planilha Excel, e as análises, realizadas através do pacote estatístico STATA 13.0. Na análise descritiva, foram apresentadas as frequências brutas e relativas e seus respectivos intervalos de confiança de 95\% (IC95\%). Para a análise bivariada, realizou-se o Teste Qui-Quadrado de Pearson, conforme pressuposto, a fim de identificar a distribuição dos sintomas depressivos pós-parto segundo as características socioeconômicas e as de apoio social. Já para a análise multivariada, utilizou-se a regressão de Poisson para verificar a associação entre a variável dependente e as variáveis independentes. As variáveis com $p<0,20$ foram incluídas na análise e a permanência se deu quando $p<0,05$. Os dados foram apresentados por meio da Razão de Prevalência (RP), bruta e ajustada com os seus respectivos IC95\%.

\section{Aspectos éticos}

O presente estudo foi aprovado pelo Comitê de Ética em Pesquisa (CAAE 69026517.2.0000.5071 - Parecer 2.149.430/2017).

\section{RESULTADOS}

A prevalência de sintomas de DPP foi de $29,7 \%$ (IC 25,0 $34,9)$ na amostra de 330 de mulheres entrevistadas no estudo (Dados não apresentados em tabela). Observa-se na Tabela 1 que $58,5 \%$ das participantes tinham idade entre 14 e 24 anos; a maioria ( $80,3 \%$ ) estava casada ou em união consensual; $64,2 \%$ possuíam escolaridade de 9 anos ou mais; e $58,2 \%$ pertenciam a classe econômica B/C.

Nota-se que $46,7 \%$ tiveram baixo apoio social material $28,5 \%$, baixo apoio afetivo; e $45,8 \%$ tiveram baixo apoio social emocional. Já o apoio de informação e de interação social positiva foi baixo em $48,2 \%$ e $45,8 \%$ (Tabela 1).

Em relação aos sintomas de DPP, as variáveis que tiveram relação significativa foram ter idade entre 14 e 24 anos (36,8\%; $p=0,001)$, estar solteira ou namorando $(44,6 \% ; p=0,003)$, ter até 8 anos de estudo $(40,7 \%$; $\mathrm{p}=0,001)$, possuir baixo apoio social material $(37,7 \% ; p=0,003)$, baixo apoio afetivo $(48,9 \%$; $p<0,001)$, baixo apoio emocional $(45 \% ; p<0,001)$, baixo apoio de informação $(40,9 \%$; $p<0,001)$ e baixa interação social positiva $(39,7 \% ; p<0,001)$ (Tabela 1).

$\mathrm{Na}$ análise multivariada, após o ajuste para as variáveis de confusão, verifica-se a associação dos sintomas de DPP com a idade, a escolaridade e o suporte social afetivo e emocional. Os sintomas de DPP foram $60 \%$ mais prevalentes em puérperas 
Tabela 1. Distribuição dos sintomas depressivos pós-parto de acordo com as características socioeconômicas e de apoio social das puérperas ( $\mathrm{n}=330$ ) internadas em uma maternidade. Cariacica, Espírito Santo, agosto a outubro de 2017

\begin{tabular}{|c|c|c|c|}
\hline Variáveis & n (\%) & Prevalência \% (IC95\%*) & p-valor \\
\hline Idade (anos) & & & 0,001 \\
\hline $14-24$ & $193(58,5)$ & $36,8(30,2-43,9)$ & \\
\hline 25 ou mais & $137(41,5)$ & $19,7(13,8-27,3)$ & \\
\hline Situação conjugal & & & 0,003 \\
\hline Casada/união consensual & $265(80,3)$ & $26,0(21,1-31,7)$ & \\
\hline Solteira/namorando & $65(19,7)$ & $44,6(32,9-56,9)$ & \\
\hline Escolaridade (anos) & & & 0,001 \\
\hline Até 8 & $118(35,8)$ & $40,7(32,1-49,8)$ & \\
\hline 9 ou mais & $212(64,2)$ & $23,6(18,3-29,8)$ & \\
\hline Classe econômica ABEP & & & 0,087 \\
\hline$B / C$ & $192(58,2)$ & $34,8(27,3-43,1)$ & \\
\hline$D / E$ & $138(41,8)$ & $26,0(20,3-32,8)$ & \\
\hline \multicolumn{4}{|l|}{ Apoio social } \\
\hline Material & & & 0,003 \\
\hline Baixo & $154(46,7)$ & $37,7(30,3-45,6)$ & \\
\hline Alto & $176(53,3)$ & $22,7(17,1-29,5)$ & \\
\hline Afetivo & & & $<0,001$ \\
\hline Baixo & $94(28,5)$ & $48,9(39,0-59,0)$ & \\
\hline Alto & $236(71,5)$ & $22,0(17,2-27,8)$ & \\
\hline Emocional & & & $<0,001$ \\
\hline Baixo & $151(45,8)$ & $45,0(37,3-53,1)$ & \\
\hline Alto & $179(54,2)$ & $16,8(11,9-23,0)$ & \\
\hline Informação & & & $<0,001$ \\
\hline Baixo & $159(48,2)$ & $40,9(33,5-48,7)$ & \\
\hline Alto & $171(51,8)$ & $19,3(14,0-25,9)$ & \\
\hline Interação social positiva & & & $<0,001$ \\
\hline Baixo & $151(45,8)$ & $39,7(32,2-47,8)$ & \\
\hline Alto & $179(54,2)$ & $21,2(15,8-27,9)$ & \\
\hline
\end{tabular}

*IC95\%: intervalo de confiança de $95 \%$.

com idade entre 14 e 24 anos em relação àquelas com idade de 25 anos ou mais ( $R P=1,60$; IC95\%: $1,10-2,34)$. Mulheres com até 8 anos de escolaridade tiveram 39\% maior prevalência de sintomas de DPP (RP=1,39; IC95\%: 1,01-2,14). As participantes com baixo apoio social afetivo e baixo apoio emocional tinham, respectivamente, 1,52 e 2,12 vezes mais prevalência de sintomas de DPP quando comparadas às que relataram alto apoio social afetivo (Tabela 2). 
Tabela 2. Análise multivariada - bruta e ajustada - das características socioeconômicas e de apoio social de puérperas ( $\mathrm{n}=330$ ) internadas em uma maternidade. Cariacica, Espírito Santo, agosto a outubro de 2017

\begin{tabular}{|c|c|c|c|c|c|c|}
\hline \multirow{2}{*}{ Variáveis } & \multicolumn{3}{|c|}{ Análise bruta } & \multicolumn{3}{|c|}{ Análise ajustada } \\
\hline & RP* & IC95\%** & $\mathrm{p}$-valor & $\mathrm{RP}^{*}$ & IC95\%** & p-valor \\
\hline Idade (anos) & & & 0,002 & & & 0,014 \\
\hline $14-24$ & 1,86 & $1,26-2,74$ & & 1,60 & $1,10-2,34$ & \\
\hline 25 ou mais & 1,0 & & & 1,0 & & \\
\hline Situação conjugal & & & 0,002 & & & 0,171 \\
\hline Casada/união consensual & 1,0 & & & 1,0 & & \\
\hline Solteira/namorando & 1,71 & $1,22-2,40$ & & 1,34 & $0,95-1,89$ & \\
\hline Escolaridade (anos) & & & 0,001 & & & 0,040 \\
\hline Até 8 & 1,72 & $1,24-2,39$ & & 1,39 & $1,01-2,14$ & \\
\hline 9 ou mais & 1,0 & & & 1,0 & & \\
\hline Classe econômica ABEP & & & 0,086 & & & \\
\hline$B / C$ & 1,0 & & & 1,0 & & 0,691 \\
\hline$D / E$ & 1,33 & $0,96-1,86$ & & 1,07 & $0,77-1,48$ & \\
\hline \multicolumn{7}{|l|}{ Apoio social } \\
\hline Material & & & 0,004 & & & 0,657 \\
\hline Baixo & 1,65 & $1,17-2,32$ & & 1,09 & $0,75-1,58$ & \\
\hline Alto & 1,0 & & & 1,0 & & \\
\hline Afetivo & & & $<0,001$ & & & 0,018 \\
\hline Baixo & 2,22 & $1,61-3,04$ & & 1,52 & $1,07-2,14$ & \\
\hline Alto & 1,0 & & & 1,0 & & \\
\hline Emocional & & & $<0,001$ & & & $<0,001$ \\
\hline Baixo & 2,69 & $1,85-3,89$ & & 2,12 & $1,41-3,19$ & \\
\hline Alto & 1,0 & & & 1,0 & & \\
\hline Informação & & & $<0,001$ & & & 0,139 \\
\hline Baixo & 2,11 & $1,47-3,03$ & & 1,36 & $0,91-2,04$ & \\
\hline Alto & 1,0 & & & 1,0 & & \\
\hline Interação social positiva & & & $<0,001$ & & & 0,716 \\
\hline Baixo & 1,87 & $1,32-2,64$ & & 1,05 & $0,72-1,52$ & \\
\hline Alto & 1,0 & & & 1,0 & & \\
\hline
\end{tabular}

*RP: razão de prevalência; **IC95\%: intervalo de confiança de $95 \%$.

\section{DISCUSSÃO}

A prevalência de sintomas de DPP no presente estudo foi de $29,7 \%$, achado semelhante ao encontrado em um serviço de saúde de São Paulo (31,2\%), ${ }^{27}$ e maior em relação a estudos conduzidos em maternidades de dois hospitais públicos de São Paulo e na Região Nordeste, nos quais a prevalência de sintomas de DPP foi de $6,7 \%{ }^{13}$ e $11,8 \%,{ }^{28}$ respectivamente. Por outro lado, há locais em que esse valor é ainda mais elevado, como no caso da Arábia Saudita, onde a prevalência sintomas de DPP foi de $57,5 \%,{ }^{29}$ e África do Sul, com $47,4 \% .{ }^{30}$ Em contrapartida, no Canadá, foi registrada uma baixa prevalência $(2,9 \%)$ de sintomas de DPP. ${ }^{31}$

No presente estudo, a alta prevalência de sintomas de DPP gera preocupação, pois a presença de sintomas depressivos pós-parto exerce influência na relação mãe-bebê, principalmente no período de três a cinco primeiros meses após o parto, pois nesse período as mães com sintomas de DPP apresentam dificuldade para desempenhar as funções maternas, manifestando sentimentos de desprezo, culpa, rejeição pela criança e raiva Outros impactos que também podem ser gerados pelos sintomas 
de DPP são: dificuldade no aleitamento materno; instabilidade no sono do bebê; ambivalência afetiva na díade; deficiência no cumprimento do calendário vacinal da criança; baixo peso; transtornos psicomotores; ${ }^{32}$ problemas de comportamento; e atrasos no desenvolvimento da linguagem desse bebê. ${ }^{6}$

Nota-se nesta pesquisa que mulheres jovens (14 a 24 anos) apresentaram maiores prevalências de sintomas de DPP, achado semelhante de um estudo realizado na Suécia, no qual avaliou 707.701 mulheres, constatando que as mais jovens e sem histórico de depressão tinham aumento significativo no risco de sintomas de DPP. ${ }^{33} \mathrm{Um}$ fator que pode explicar esse achado é que mães mais jovens estão mais expostas a situações de vulnerabilidades, preocupações e estresse. ${ }^{34}$ Puérperas jovens experimentam desafios adicionais, por estarem em uma fase de desenvolvimento de suas próprias vidas, tendo que, muitas vezes, abrir mão de metas e desejos, devido às responsabilidades de cuidar de um recém-nascido. ${ }^{33}$

Mulheres com menor escolaridade apresentaram 39\% maior prevalência de sintomas de DPP, achado semelhante ao encontrado em estudo transversal uma maternidade de referência no Paraná em 2015. ${ }^{35}$ Estudo realizado no município de Rio Grande (RS), com 2.687 mulheres, evidenciou a relação entre escolaridade e maior frequência de DPP, uma vez que, quanto maior os anos completos de estudo, maior a proteção no desenvolvimento de depressão. ${ }^{16} \mathrm{~A}$ educação é um componente que influencia e ajuda no gerenciamento efetivo de problemas. Destaca-se que a baixa escolaridade interfere na habilidade da puérpera de agir frente aos problemas impostos pela maternidade, podendo levar a mulher ao sentimento de insegurança em relação à sua capacidade de ser mãe. ${ }^{36}$

Culturalmente, a gravidez é vista como um momento de felicidade e de expectativa na vida de muitas mulheres, considerando a perspectiva de alegria que um novo filho trará para a família. Ao mesmo tempo, a gestação pode ser um evento estressante e que provoca ansiedade, e algumas mulheres podem vivenciar problemas de saúde mental perinatal neste período, principalmente devido à grande preocupação com a saúde de seu filho, com a capacidade de cuidar da criança e ser uma boa mãe.$^{37}$ Além disso, a idade jovem e a falta de oportunidade de alcançar um nível educacional mais alto podem aumentar a vulnerabilidade social e econômica e acentuar a ansiedade com a chegada do bebê. ${ }^{34}$ Uma gestação também pode estar relacionada a uma gravidez indesejada, o que acentua a probabilidade de sintomas de DPP. ${ }^{38}$

O achado de maior prevalência de sintomas depressivos pós-parto em puérperas com menor apoio social emocional deste estudo faz coro com os resultados encontrados em uma pesquisa realizada em uma clínica obstétrica na Polônia, onde as pacientes com maior risco de apresentar sintomas de DPP obtiveram menores escores de apoio social emocional. ${ }^{39} \mathrm{O}$ apoio emocional permite que a puérpera expresse seus sentimentos frente à maternidade, como a insegurança, os sentimentos de inadequação e expectativas quanto ao bebê ou ao seu desempenho como mãe. A falta desse apoio nesse período pode causar sofrimento emocional. ${ }^{15}$

Ainda, este estudo observou uma associação entre baixo apoio social afetivo com uma maior prevalência de sintomas de DPP, uma vez que o indivíduo que apresenta baixo apoio social afetivo pode estar envolvido em um ciclo social com disfunções nas demonstrações físicas de amor e afeto. ${ }^{40}$ Mulheres que recebem apoio social afetivo têm uma melhor percepção de seu estado de saúde, o que proporciona um melhor discernimento do auxílio que recebe de sua matriz de suporte. ${ }^{41}$

Estudo realizado em uma maternidade pública de São Paulo evidenciou que $23 \%$ das mulheres pertencentes ao grupo de risco de sintomas de DPP recebiam qualquer tipo de apoio social afetivo e emocional. ${ }^{42} \mathrm{Em}$ relação à ajuda de amigos e familiares no cuidado da criança, $80 \%$ das puérperas não pertencentes ao grupo de risco de sintomas de DPP recebiam essa ajuda, enquanto esse suporte de amigos e familiares foi menos presente (38\%) nas mães do grupo de risco. Além disso, aquelas que apresentaram maiores pontuações para sintomas de DPP eram aquelas com pouco suporte familiar e psicológico. ${ }^{42}$ Cuidar do recém-nascido requer conhecimento, dedicação, paciência e disposição. Nesse cenário, algumas mulheres, por inexperiência e/ou insegurança, não conseguem realizar tais cuidados, necessitando de ajuda até sua adaptação. ${ }^{43}$

As limitações do estudo consistem no fato dos dados aqui apresentados serem coletados em uma maternidade pública, de modo a não ser possível generalizar os achados para a população total de puérperas, todavia vale destacar que está é a única maternidade pública do município. Outro ponto a mencionar é a possibilidade do viés de informação, contudo a entrevista ocorreu em local privativo após a explicação do objetivo da pesquisa e o aceite da participante, assim como também a utilização de instrumentos validados para rastreio dos agravos, sendo fatores que contribuem na redução desse viés.

\section{CONCLUSÃO E IMPLICAÇÕES PARA A PRÁTICA}

Por fim, o estudo revela uma alta prevalência de sintomas de DPP entre as participantes e essa prevalência está relacionada com características socioeconômicas e de apoio social, sendo elas: puérperas jovens (14-24 anos), baixo nível de suporte social afetivo e baixo nível emocional durante a gestação.

Nesse cenário, os profissionais inseridos nos serviços de saúde devem promover o acompanhamento em saúde mental das puérperas, que pode ser iniciado principalmente no próprio pré-natal através das consultas, a fim de detectar os fatores de risco em gestantes com potencial de desenvolver os sintomas depressivos pós-parto. Ainda, cabe destacar a importância da continuidade da assistência às mulheres no puerpério com a oferta de atendimento qualificado e multidisciplinar, integrando as diferentes áreas do cuidado. Desse modo, faz-se necessário a atuação da equipe de saúde no acolhimento, detecção e tratamento da DPP. Visto que é uma doença psíquica os 
psicólogos, psiquiatras e obstetras podem trabalhar em conjunto, a fim de ofertar assistência integral à mulher e à sua família nos serviços de saúde.

Assim, os profissionais da atenção primária possuem um papel essencial, visto que, por vezes, estão presentes no cuidado à mulher durante todo o período gravídico-puerperal. Esse contato com a mulher favorece a construção de vínculo, no qual cria-se um espaço oportuno para a puérpera compartilhar as inquietudes e angústias frente às dificuldades, tornando possível o desenvolvimento de um plano de cuidados de acordo com as necessidades de cada mulher.

\section{CONTRIBUIÇÕES DOS AUTORES}

Desenho do estudo. Franciéle Marabotti Costa Leite.

Coleta ou produção dos dados. Franciéle Marabotti Costa Leite. Maria Luiza Cunha Santos.

Análise de dados. Franciéle Marabotti Costa Leite. Dherik Fraga Santos. Ranielle de Paula Silva. Joyce Ferreira Reis. Maria Luiza Cunha Santos.

Interpretação dos resultados. Franciéle Marabotti Costa Leite. Dherik Fraga Santos. Ranielle de Paula Silva. Joyce Ferreira Reis.

Redação e revisão crítica do manuscrito. Maria Luiza Cunha Santos. Joyce Ferreira Reis. Ranielle de Paula Silva. Dherik Fraga Santos. Franciéle Marabotti Costa Leite.

Aprovação da versão final do artigo. Maria Luiza Cunha Santos. Joyce Ferreira Reis. Ranielle de Paula Silva. Dherik Fraga Santos. Franciéle Marabotti Costa Leite.

Responsabilidade por todos os aspectos do conteúdo e a integridade do artigo publicado. Maria Luiza Cunha Santos. Joyce Ferreira Reis. Ranielle de Paula Silva. Dherik Fraga Santos. Franciéle Marabotti Costa Leite.

\section{EDITOR ASSOCIADO}

Candida Primo Caniçali $\odot$

\section{EDITOR CIENTÍFICO}

\author{
Ivone Evangelista Cabral (B)
}

\section{REFERÊNCIAS}

1. Organização Pan-Americana de Saúde. Folha Informativa: depressão [Internet]. Brasília: Organização Pan-Americana de Saúde; 2018. [citado 2021 mai 05]. Disponível em: https://www.paho.org/pt/topicos/ depressao.

2. Santos RNC, Bellato R, Araújo LFS, Almeida KBB, Souza IP. Lugares do homem no cuidado familiar no adoecimento crônico. Rev Esc Enferm USP. 2018;52:e03398. http://dx.doi.org/10.1590/s1980-220x2017046703398. PMid:30540127.

3. Ministério da Saúde (BR). Depressão: causas, sintomas, tratamentos, diagnóstico e prevenção [Internet]. Brasília: Ministério da Saúde;2020a. [citado 2021 mai 18]. Disponível em: https://antigo.saude.gov.br/saudede-a-z/depressao.

4. American Psychiatric Association. Manual diagnóstico e estatístico de transtornos mentais. 5ª edição. Porto Alegre: Artmed; 2014.
5. Martins AB, Ribeiro J, Soler ZASG. Proposta de exercícios físicos no pós-parto. Um enfoque na atuação do enfermeiro obstetra. Invest Educ Enferm. 2011;29(1):40-5

6. Ministério da Saúde (BR). Depressão pós-parto: causas, sintomas, tratamento, diagnóstico e prevenção. [Internet]. Brasília: Ministério da Saúde; 2020b. [citado 2021 mai 01]. Disponível em: https://antigo. saude.gov.br/saude-de-a-z/saude-mental/depressao-pos-parto.

7. Secretaria de Estado de Saúde de Goiás. Depressão pós-parto. [Internet] Goiânia: Secretaria de Estado de Saúde de Goiás;2019. [citado 2021 jun 01]. Disponivel em: https://www.saude.go.gov.br/biblioteca/7594depress\%C3\%A3o-p\%C3\%B3s-parto.

8. Greinert BRM, Milani RG. Depressão Pós-Parto: Uma Compreensão Psicossocial. Psicol Teor Prat. 2015;17(1):26-36. http://dx.doi. org/10.15348/1980-6906/psicologia.v17n1p26-36

9. Cepeda MS, Kern DM, Nicholson S. Treatment resistant depression in women with peripartum depresson. BMC Pregnancy Childbirth. 2019;19:323. http://dx.doi.org/10.1186/s12884-019-2462-9.

10. Fiala A, Svancara J, Klanova J, KasparekT. Sociodemographic and delivery risk factors for developing postpartum depression in a sample of 3233 mothers from the Czech ELSPAC study. BMC Psychiatry. 2017;17(1):104. http://dx.doi.org/10.1186/s12888-017-1261-y. PMid:28327118.

11. Salem MD, Thabet MD, Fouly $H$, Abbas AM. Factors affecting the occurrence of postpartum depression among puerperal women in Sohag city, Egypt. Obstet Gynecol. 2017;7(1):4. http://dx.doi.org/10.17077/21544751.1328.

12. Rojas G, Guajardo V, Martínez P, Fritsch R. Depresión posparto: tamizaje, uso de servicios y barreras para su tratamiento en centros de atención primaria. Rev Med Chil. 2018;146(9):1001-7. http://dx.doi.org/10.4067/ s0034-98872018000901001. PMid:30725020.

13. Poles MM, Carvalheira APP, Carvalhaes MABL, Parada CMGL. Sintomas depressivos maternos no puerpério imediato: fatores associados. Acta Paul Enferm. 2018;31(4):351-8. http://dx.doi.org/10.1590/19820194201800050.

14. Salgado HO. Cuidado materno livre de danos e prevalência de depressão pós-parto [tese]. São Paulo: Universidade de São Paulo;2017 [citado 2021 mai 15]. Disponível em: https://www.teses.usp.br/teses/disponiveis/6/6136/ tde-02082017-173259/publico/HeloisaDeOliveiraSalgadoSIMPLIFICADA pdf.

15. Almeida NMC, Arrais ARO. Pré-natal psicológico como programa de prevenção à depressão pós-parto. Psicologia. 2016;36(4):847-63. http:// dx.doi.org/10.1590/1982-3703001382014.

16. Hartmann JM, Mendoza-Sassi RA, Cesar JA. Depressão entre puérperas: prevalência e fatores associados. Cad Saude Publica. 2017;33(9):e00094016. http://dx.doi.org/10.1590/0102-311x00094016. PMid:29019521.

17. Valla VV. Educação popular, saúde comunitária e apoio social numa conjuntura de globalização. Cad Saúde Pública. 1999;15(suppl.2):7-14 http://dx.doi.org/10.1590/S0102-311X1999000600002.

18. Pietrukowicz MCLC. Apoio social e religião: Uma forma de enfrentamento dos problemas de saúde [dissertação]. Rio de Janeiro: Escola Nacional de Saúde Pública; 2001 [citado 2021 jun 02]. Disponível em: https:// www.arca.fiocruz.br/bitstream/icict/4610/2/213.pdf.

19. Mlotshwa L, Manderson L, Merten S. Personal support and expressions of care for pregnant women in Soweto, South Africa. Glob Health Action. 2017;10(1):1363454. http://dx.doi.org/10.1080/16549716.2017.1363 454. PMid:28874098

20. Avanzi SA, Dias CA, Silva LOL, Brandão MBF, Rodrigues SM. Importância do apoio familiar no período gravídico-gestacional sob a perspectiva de gestantes inseridas no PHPN. Rev Saúde Col. 2019;9(55):55-62. http://dx.doi.org/10.13102/rscdauefs.v9i0.3739.

21. Costa JF. Depressão pós-parto: a atuação do enfermeiro na identificação precoce da doença [dissertação]. Itabuna: União Metropolitana de Educação e Cultura; 2017 [citado 2021 jun 10]. Disponível em: https://repositorio.pgsskroton.com/bitstream/123456789/21058/1/ JANINE_FERREIRA_COSTA_ATIVIDADE\%2b4.pdf.

22. Instituto Brasileiro de Geografia e Estatística. Cidades e Estados: Cariacica [Internet]. Rio de Janeiro: IBGE; 2019 [citado 2021 mai 02] Disponível em: https://www.ibge.gov.br/cidades-e-estados/es/cariacica. html. 
23. Oliveira LCQ, Fonseca-Machado MO, Stefanello J, Gomes-Sponholz FA. Intimate partner violence in pregnancy: identification of women victims of their partners. Rev Gaúcha Enferm. 2015;36(Spec No):233-8. http:// dx.doi.org/10.1590/1983-1447.2015.esp.57320. PMid:27057724.

24. Ribeiro JLP, Ponte ACSLC. Propriedades métricas da versão portuguesa da escala de suporte social do MOS (MOS Social Support Survey) com idosos. Psicol Saúde Doenças. 2009;10(2):163-74.

25. Griep RH, Chor D, Faerstein E, Werneck GL, Lopes CS. Validade de constructo de escala de apoio social do Medical Outcomes Study adaptada para o português no Estudo Pró-Saúde. Cad Saude Publica. 2005;21(3):703-14. http://dx.doi.org/10.1590/S0102-311X2005000300004 PMid:15868028.

26. Cox JL, Holden JM, Sagovsky R. Detection os postnatal depression: development of the 10-item Edinburgh postnatal depression scale. $\mathrm{Br} \mathrm{J}$ Psychiatry. 1987;150(6):782-6. http://dx.doi.org/10.1192/bjp.150.6.782. PMid:3651732.

27. Abuchaim ESV, Caldeira NT, Lucca MMD, Varela M, Silva IA. Depressão pós-parto e autoeficácia materna para amamentar: prevalência e associação. Acta Paul Enferm. 2016;29(6):664-70. http://dx.doi. org/10.1590/1982-0194201600093.

28. Silva CS, Lima MC, Sequeira-de-Andrade LAS, Oliveira JS, Monteiro JS, Lima NMS et al. Association between postpartum depression and the practice of exclusive breastfeeding in the first three months of life. $J$ Pedriatr. 2017;93(4):356-64. http://dx.doi.org/10.1016/j.jped.2016.08.005. PMid:28034730.

29. Bawahab JA, Alahmadi JR, Ibrahim AM. Prevalence and determinants of antenatal depression among women attending primary health care centers in Western Saudi Arabia. Saudi Med J. 2017;38(12):1237-42. http://dx.doi.org/10.15537/smj.2017.12.21262. PMid:29209674.

30. Dlamini LP, Mahanya S, Dlamini SD, Shongwe MC. Prevalence and factors associated with postpartum depression at a primary healthcare facility in Eswatini. S Afr J Pshychiatr. 2019;25(1):1404.

31. Dennis CL, Merry L, Gagnon AJ. Postpartum depression risk factors among recent refugee, asylum-seeking, non-refugee immigrant, and Canadian-born women: results from a prospective cohort study. Soc Psychiatry Psychiatr Epidemiol. 2017;52(4):411-22. http://dx.doi. org/10.1007/s00127-017-1353-5. PMid:28220215.

32. Greinert BRM, Carvalho ER, Capel H, Marques AG, Milani RG. A relação mãe-bebê no contexto da depressão pós-parto: estudo qualitativo. Revista Saúde e Pesquisa. 2018;11(1):81-8. http://dx.doi. org/10.17765/1983-1870.2018v11n1p81-88.
33. Silverman ME, Reichenberg A, Savitz DA, Cnattingius S, Lichtenstein P, Hultman $\mathrm{CM}$ et al. The risk factors for postpartum depression: a population-based study. Depress Anxiety. 2017;34(2):178-87. http:// dx.doi.org/10.1002/da.22597. PMid:28098957.

34. Muraca GM, Joseph KS. The association between maternal age and depression. J Obstet Gynaecol Can. 2014;36(9):803-10. http://dx.doi. org/10.1016/S1701-2163(15)30482-5. PMid:25222359.

35. Lima NC, Ravelli APX, Messias LSF, Skupien SV. Depressão pósparto baseada na escola de Edimburgo. Revista Conexão UEPG. 2016;12(2):268-77. http://dx.doi.org/10.5212/Rev.Conexao.v.12. i2.0008.

36. Mazaheri MA, Rabiei L, Masoudi R, Hamidizadeh S, Nooshabadi MRR Najimi A. Understanding the factors affecting the postpartum depression in the mothers of Isfahan City. J Educ Health Promot. 2014;3:65. http:// dx.doi.org/10.4103/2277-9531.134784. PMid:25077158.

37. Islam J, Broidy L, Baird K, Mazerolle P. Intimate partner violence around the time of pregnancy and postpartum depression: The experience of women of Bangladesh. PLoS One. 2017;12(5):e0176211. http://dx.doi. org/10.1371/journal.pone.0176211. PMid:28472056.

38. Tikmani SS, Soomro T, Tikmani P. Prevalence and determinants of postpartum depression in a tertiary care hospital. Austin J Obstet Gynecol. 2016;3(2):1057.

39. Maliszewska K, Swiatkowska-Freund M, Bidzan M, Preis K. Relationship, social support, and personality as psychosocial determinants of the risk for postpartum blues. Ginekol Pol. 2016;87(6):442-7. http://dx.doi. org/10.5603/GP.2016.0023. PMid:27418222.

40. Matias RC, Martinelli SC. Um estudo correlacional entre apoio social e autoconceito de estudantes universitários. Avaliação;2017;22(1):15-33. http://dx.doi.org/10.1590/s1414-40772017000100002

41. Frizzo GB, Martins LWF, Silva EXL, Piccinini CA, Diehl AMP. Maternidade adolescente: a matriz de apoio e o contexto de depressão pós-parto. Psicol, Teor Pesqui. 2019;35:e3533. http://dx.doi.org/10.1590/0102.3772e3533.

42. Andrade ALM, Teixeira LRS, Zoner CC, Niro NN, Scatena A, Amaral RA. Fatores associados à depressão pós-parto em mulheres em situação de vulnerabilidade social. Eletrônica Saúde Mental Álcoo Drog. 2018;13(4):196-204. http://dx.doi.org/10.11606/issn.1806-6976. v13i4p196-204.

43. Cremonese L, Wilhelm LA, Prates LA, Paula CC, Sehnem GD, Resse LB. Apoio social na perspectiva da puérpera adolescente. Esc Anna Nery. 2017;21(4). http://dx.doi.org/10.1590/2177-9465-EAN-2017-0088.

\footnotetext{
a Artigo derivado do projeto de pesquisa "Violência contra a mulher na gestação e a depressão pós-parto: estudo em uma maternidade de baixo risco", financiado pela Fundação de Amparo à Pesquisa e Inovação do Espírito Santo (FAPES). - Edital FAPES/CNPq 04/2017. Processo 80641393/2017, concedido a Franciéle Marabotti Costa Leite.
} 